\title{
A THREE LAYER MODEL FOR THE THERMAL IMPEDANCE OF THE HUMAN SKIN: MODELING AND EXPERIMENTAL MEASUREMENTS
}

\author{
MARIA STRA̧KOWSKA*, *, GILBERT DE MEY ${ }^{\dagger, \S, ~}$ \\ BOGUSŁAW WIECCEK ${ }^{*, \uparrow}$ and MICHAE STRZELECKI*, \\ *Institute of Electronics, Lódź University of Technology \\ Wólczańska str., 211-215 \\ 90-924 Lódź, Poland \\ $\dagger$ Electronics and Information Systems Dept., Ghent University \\ Sint Pietersnieuwstraat 41 \\ 9000 Ghent, Belgium \\ ^strakowska.m@gmail.com \\ §gilbert.demey@ugent.be \\ Iwiecek@p.lodz.pl \\ ${ }{ }^{\text {michal.strzelecki@p.lodz.pl }}$
}

Received 19 August 2014

Revised 24 September 2014

Accepted 25 September 2014

Published 13 January 2015

\begin{abstract}
In this paper a dynamic three layer model for the heat transfer in the human skin is presented. The model is solved in the Laplace domain using the phasor notation. In order to compare the theoretical model with experimental results, a transient heating was carried out and the time dependent skin temperature was recorded with a thermographic camera. The transient temperature could be fitted very well to an analytical function, which could easily be transformed into the Laplace domain allowing an easy comparison between the model and the experimental results. The aim of the research is to evaluate the skin thermal parameters for all layers including the blood perfusion.
\end{abstract}

Keywords: IR thermography; heat transfer modeling; perfusion, thermal impedance.

\section{Introduction}

The human skin contains three layers: epidermis, dermis and hypodermis. Epidermis is the thinnest layer and there are no blood vessels in it. Dermis contains blood and lymphatic vessels, nerves, sweat glands and sebaceous. There are blood vessels and nerves within hypodermis. The thickness of this layer depends on the part of the body and the amount of fat. It is the intention of this paper to measure the thermal properties of these layers in a non-invasive way by using infrared 
thermography after an external skin cooling was applied. It is a kind of functional imaging based on so-called cold stress method well-known in medicine. ${ }^{1,2}$

Thermal modeling of a human tissue with the blood perfusion is known in the literature $^{3-10}$ and it is still an important research domain. Nowadays, the inverse thermal modeling can be applied for evaluating the thermal parameters of the skin. The thermal modeling of the tissue significantly differs from the heat transfer in solids and fluids. One has to consider the perfusion and the blood flow. The tissue is a non-homogenous and anisotropic body. It makes the solution of the model presented by a set of differential equations more complicated. The attempt of including the perfusion in thermal modeling was presented by Pennes in 1948. ${ }^{3,4,8}$ Chen and Holmes (1980) proposed modeling the heat transfer in a tissue as in the porous material. ${ }^{5}$ They divided a tissue into two parts: solid-like and fluid-like ones. They used blood (fluid) velocity $(v, m / s)$ to model the additional heat transfer due to the blood movement. They proposed a simple way to split the model into the solid and fluid parts using the partitioning factor. ${ }^{5}$ In addition, the Chen-Holmes model assumes the heat transfer between the blood vessels and the solid tissue. The main problem in this approach is how to define the velocity of blood and the partitioning factor. Weinbaum, Jiji and Lemons (1984) proposed a model including the heat transfer between a tissue and arteries and veins. ${ }^{6}$ They assume that the arteries and veins are typically parallel to each other, and a significant amount of heat is transferred between them. The simplified model published by Weinbaum and $\mathrm{Jiji}^{7}$ uses a so-called effective thermal conductivity $k_{\text {eff }}$ and geometry factor which takes into account the three-dimensional heat transfer in the tissue. There are numerous analytical and numerical models for heat transfer in the tissue. ${ }^{8,9,11}$ Some of them need to be calibrated with the measuring data, because they use many parameters with explicitly unknown values.

Infrared thermography has been used to investigate many skin and other tissue pathologies. For example, thermography was applied for burn injuries, ${ }^{12,13}$ ocular surface temperature measurement, ${ }^{14}$ breast tumors, ${ }^{10,15}$ and skin heating by radiation. ${ }^{10}$

In this research, three layers of the skin tissue are considered: dermis, epidermis and hypodermis and the Pennes model is used for the perfusion. ${ }^{3,8}$ For all of them the blood perfusion and thermal conduction in the transient state is taken into account. At the skin surface, convection cooling to the ambient air is also modeled. It must be pointed out here that both the model and the experiments are carried out in a dynamic way. It means that all investigations are either time dependent or performed in the frequency domain, taking the advantage of using complex thermal impedance. ${ }^{16-18}$ This will allow us to put more emphasis on the higher frequencies which corresponds to short time intervals. The influence due to varying ambient conditions like room temperature or air velocity will then have a minor influence. In order to eliminate the influence of body movement during the measurements, a specially developed software for movement correction was applied to correct the thermographic images. ${ }^{2}$ 
In other fields, like electrical engineering and electronics, the idea of thermal impedance gained a lot of interest during the last two decades. Thermal impedances have been measured or calculated in integrated circuits, electronic packages and high voltage cables. ${ }^{16,17,19}$ The thermal impedance can be represented in many ways, just like electric impedances. In this paper the Nyquist plot representation of the measured date is used. ${ }^{16,19}$ Other data like the thermal time constant distribution, the integral and the differential structure function can be useful as well. ${ }^{19}$ Their behavior at different frequencies provides us much more information than a simple thermal resistance or a temperature distribution in steady state conditions.

\section{Three Layer Thermal Model of the Skin}

As it has already been mentioned, the skin is composed of three layers called dermis, epidermis and hypodermis having thicknesses $d_{1}, d_{2}$ and $d_{3}$, respectively as shown schematically in Fig. 1. The heat flow is assumed to be purely one-dimensional, i.e., heat only flows in the $x$-direction perpendicular to the skin's surface. Each layer has a thermal conductivity $k_{i}$, a density $\varrho_{i}$ and a specific heat per unit weight $c_{i}$ $(i=1, \ldots, 3)$. The governing equation for the temperature distributions $T_{i}(x, t)$ for $i=1, \ldots, 3$ are then:

$$
k_{i} \frac{\partial^{2} T_{i}}{\partial x^{2}}=c_{i} \varrho_{i} \frac{\partial T_{i}}{\partial t}-w_{i} c_{b} \varrho_{b}\left(T_{b}-T_{i}\right) .
$$

The quantity $c_{b}$ denotes the specific heat per unit weight, $\varrho_{b}$ the density and $T_{b}$ the temperature of the blood. The coefficient $w_{i}$ describes the perfusion rate. Physically the expression $w_{i} c_{b} \varrho_{b}\left(T_{b}-T_{i}\right)$ describes the amount of heat transferred from the blood to a tissue layer at a temperature $T_{i}$. For the first layer the blood perfusion can usually be neglected. Quantities $k_{i}, c_{i}$ and $\varrho_{i}$ represent the thermal conductivity, the specific heat per unit weight and the density of the $i$ th layer.

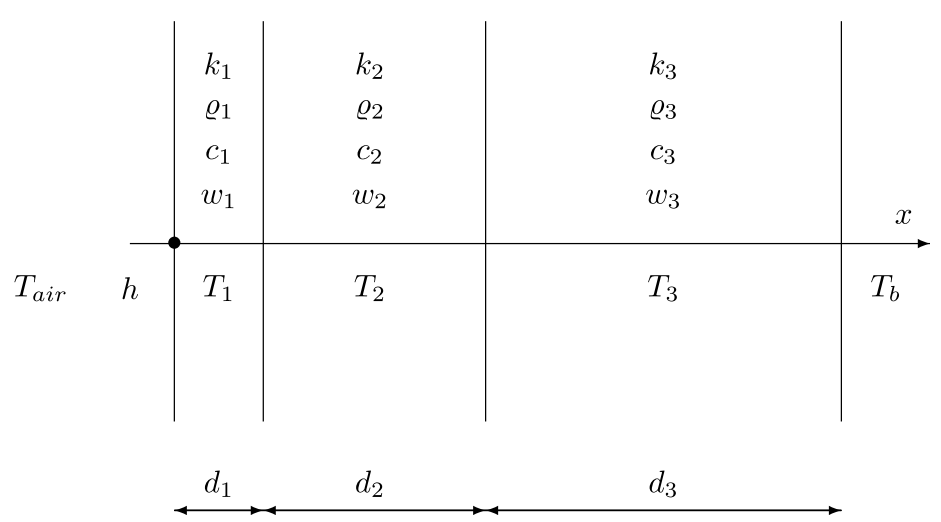

Fig. 1. Three layer model for the skin. 
In order to solve the Eq. (1), suitable boundary conditions are needed. At the two interfaces one has the continuity of the temperature and the heat flux.

$$
\begin{aligned}
T_{1}\left(d_{1}, t\right) & =T_{2}\left(d_{1}, t\right), \\
T_{2}\left(d_{1}+d_{2}, t\right) & =T_{3}\left(d_{1}+d_{2}, t\right), \\
k_{1}\left(\frac{\partial T_{1}}{\partial x}\right)_{d_{1}} & =k_{2}\left(\frac{\partial T_{2}}{\partial x}\right)_{d_{1}}, \\
k_{2}\left(\frac{\partial T_{2}}{\partial x}\right)_{d_{1}+d_{2}} & =k_{3}\left(\frac{\partial T_{3}}{\partial x}\right)_{d_{1}+d_{2}} .
\end{aligned}
$$

At the skin surface $x=0$ convection to the ambient air is taken into account.

$$
h\left[T_{1}(0, t)-T_{\text {air }}\right]=k_{1}\left(\frac{\partial T_{1}}{\partial x}\right)_{x=0}+P(t) .
$$

The parameter $P(t)$ is the power flux $\left(W / m^{2}\right)$ applied to the skin in order to induce a temperature change. As it will be outlined later on in the experimental section, we did not apply a power flux at the skin surface but a previous cooling. Hence $P(t)$ has to be interpreted as the equivalent power flux which gives rise to the same transient phenomenon.

At the rightmost point, for $x=d_{1}+d_{2}+d_{3}$ the temperature is assumed to be constant and equal to the body's blood temperature $T_{b}$ :

$$
T_{3}\left(d_{1}+d_{2}+d_{3}, t\right)=T_{b} .
$$

The boundary conditions (2)-(7) are sufficient to solve Eq. (1).

Equation (1) cannot be solved analytically in the time domain. In the Laplace domain ( $s$ variable) or the frequency domain $(s \rightarrow j \omega)$ the equations are much easier to deal with. In this paper we will focus only on the frequency domain analysis also known as the AC analysis in the field of electrical engineering. It is also assumed that the AC signals (temperatures, fluxes) are all superposed on the DC or the steady state values. In this paper steady state values are not evaluated, only the alternating $\mathrm{AC}$ components superposed on it. In the frequency domain Eq. (1) is changed into $(\partial / \partial t \rightarrow j \omega)$ :

$$
\begin{gathered}
k_{i} \frac{d^{2} \Delta T_{i}}{d x^{2}}=j \omega \varrho_{i} c_{i} \Delta T_{i}+w_{i} c_{b} \varrho_{b} \Delta T_{i}, \\
\frac{d^{2} \Delta T_{i}}{d x^{2}}=\frac{\Delta T_{i}}{L_{i}^{2}}
\end{gathered}
$$

where for all the layers, the complex diffusion length is defined by:

$$
L_{i}=\frac{1}{\sqrt{\frac{j \omega \varrho_{i} c_{i}}{k_{i}}+\frac{w_{i} c_{b} \varrho_{b}}{k_{i}}}} .
$$


To avoid any confusion, AC components is denoted by $\Delta$. Remark that the constant blood temperature $T_{b}$ seems to be disappeared from (8) and (11). This is due to the fact that all the AC temperatures to be calculated from (1) have to be superposed on the steady state temperature distribution, including $T_{b}$. The boundary conditions remain almost unchanged except for the fact that the partial derivative $\partial / \partial x$ can be replaced by $d / d x$. A minor change has to be made to the boundary condition (6). Because $T_{\text {air }}$ is a constant temperature, Eq. (6) is replaced by:

$$
h \Delta T_{1}(0)=k_{1}\left(\frac{\partial \Delta T_{1}}{\partial x}\right)_{x=0}+P_{\mathrm{AC}},
$$

where $P_{\mathrm{AC}}$ is the $\mathrm{AC}$ component of the power flux applied at the surface.

The general solution of the Eq. (1) can then be written as:

$$
\Delta T_{i}=A_{i} e^{-x / L_{i}}+B_{i} e^{x / L_{i}} .
$$

Filling in the six boundary conditions (2)-(5), (7) and (11) gives rise to an algebraic set of six equations with six unknowns: $A_{i}$ and $B_{i}(i=1, \ldots, 3)$. Using (12) for $x=0$ and $i=1$ gives us the surface skin temperature which is also measured experimentally using thermography.

With the three layer model it is rather straightforward to solve the system of equations and boundary conditions in the complex or the Laplace domain. Meanwhile, the experimental measurements are carried out in the time domain, i.e., a transient temperature recording using the infrared thermography. Therefore the procedure applied in this paper is the following.

- First of all the transient skin temperature is measured.

- These experimental data are fitted to an analytic function of time in order to eliminate the noise of the temperature recording.

- This analytic function of time can be exactly transformed into the Laplace domain. This gives rise to a Nyquist plot of the skin temperature.

- The skin temperature obtained by solving the three layer model is fitted with the Nyquist plot.

- From this fitting, numerical values for all parameters $\left(k_{i}, w_{i}, c_{i}\right.$ and $\left.h\right)$ are obtained.

In the subsequent sections, these points will be outlined in detail.

\section{Experimental Measurements}

Several types of skin cooling were considered such as cooling gel taken from the fridge or blowing compressed air from a certain distance. The latter one turned out to be better, due to a more uniform cooling of the skin area. After this cooling period, the thermographic measurements were started. A CEDIP Titanium thermographic camera was used. This camera has a matrix of $640 \cdot 512, \operatorname{In} S b$ detectors, 
which are cooled to cryogenic temperatures using a built in Stirling cooler. Microbolometer cameras cannot be used in this case, because from time to time a shutter blocks the image recording followed by non-uniformity correction which can give rise to discontinuities in the temperature readout. Because the person (patient) under investigation is breathing and moving, a movement correction was implemented using a technique based on the cross correlation method. ${ }^{2}$ It requires that a few marks have to be put on the skin. These marks were made from thin aluminium foils which have a very low emissivity so that they were easily visible in the thermographic images.

For the modeling it was found that the AC or phasor notation turned out to be the most suitable approach because the time dependent analysis did not allow an analytical solution for more than one layer. For the experimental investigation only transient measurements are possible because alternating heat sources with variable frequency are very difficult or even impossible to make. In our experiments, a cold pack was put on the skin for a sufficiently long time. When the cold pack was removed, the skin temperature was measured with an infrared thermographic camera as a function of time till steady state was obtained. A typical example of such measurements is shown as a thermal image and temperature-versus-time curve in Figs. 2 and 3. Note that in Fig. 3 we put $T=0$ as the initial temperature, just as a reference. In the beginning the temperature rise is very sharp similar to the $\sqrt{t}$ heating reported in many papers related to surface heating or cooling. ${ }^{20,21}$ After some time the curve has more the character of an exponential function. After a sufficient long time, the steady state value of $1.5^{\circ} \mathrm{C}$ is obtained which corresponds to the normal skin temperature.

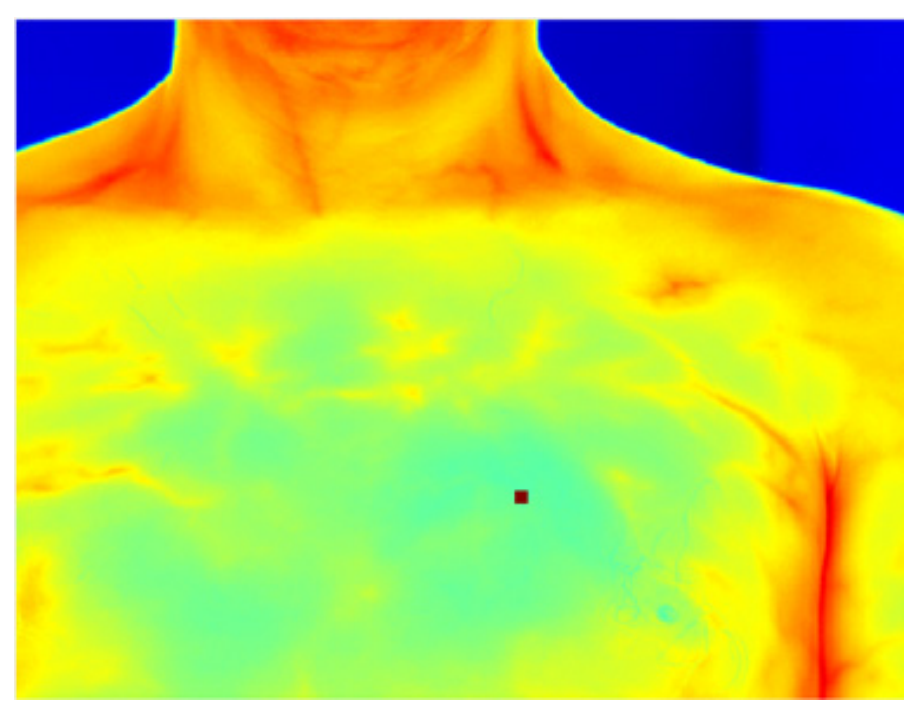

Fig. 2. Thermographic image of a skin (one of a sequence). 


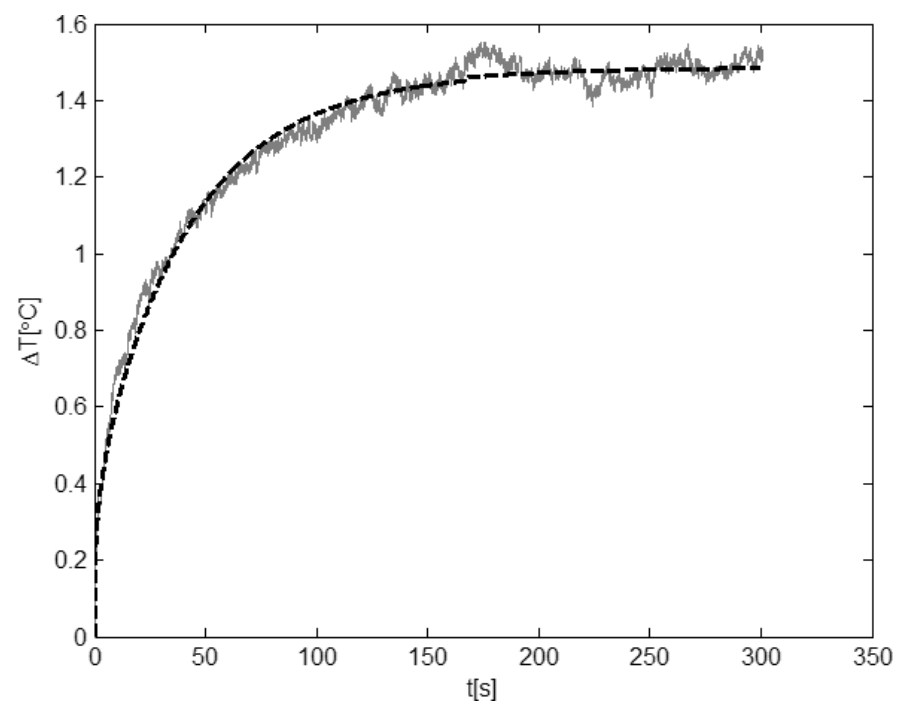

Fig. 3. Transient temperature recordings of the skin after removal of the cold pack and its smoothing approximation.

The experimental curve shown in Fig. 3 could be very well fitted to the following analytical function:

$$
f(t)=A\left[1-e^{-t / \tau_{0}}\right]+B\left[1-e^{t / \tau_{1}} \operatorname{erfc}\left(\sqrt{t / \tau_{1}}\right)\right],
$$

where erfc denotes the complementary error function. ${ }^{23}$ If $t \rightarrow \infty$ the function (13) approaches a constant value given by:

$$
f(\infty)=A+B
$$

For small values of the time $t$, the function (13) can be approximated by:

$$
f(t)=2 B \sqrt{\frac{t}{\pi \tau_{1}}} .
$$

Using $A=1.0, B=0.5, \tau_{0}=43.47 \mathrm{~s}$ and $\tau_{1}=1.666 \mathrm{~s}$, the fitting function (13) has also been plotted in Fig. 3. As one can observe the agreement with the experimental recording is quite satisfactory. The function (13) was used because both terms are well-known solutions for transient thermal problems. The exponential term is a transient temperature of a good thermal conductor uniformly heated and subjected to convective cooling. The second term with the erfc function is transient solution for an half infinite $1 D$ medium with convective cooling at the front surface. ${ }^{22}$

The comparison between the experimental data and the fitted curve shows a maximum discrepancy of $0.1^{\circ} \mathrm{C}$ around $t=180 \mathrm{~s}$. Referring to the maximum temperature of $1.5^{\circ} \mathrm{C}$, it corresponds roughly to an uncertainty of $0.1 / 1.5=6.6 \%$.

The analytical function (13) has not only the advantage of a good fit to the experimental data. The function (13) allows an analytic evaluation of the 
corresponding thermal impedance in the frequency domain. From the step response (12), the impedance $F(j \omega)$ can be evaluated by the integral ${ }^{19}$ :

$$
F(j \omega)=j \omega \int_{0}^{\infty} e^{-j \omega t} f(t) d t
$$

By replacing $j \omega$ by the Laplace variable $s$, one can evaluate the integral by searching in a table of Laplace transforms. ${ }^{23}$ The final result is then:

$$
F(j \omega)=\frac{A}{1+j \omega \tau_{0}}+\frac{B}{1+\sqrt{j \omega \tau_{1}}} .
$$

Figure 4 shows a representation of (17). It is a so called Nyquist plot, representing the imaginary part $\operatorname{Im}\{F(j \omega)\}$ versus the real part $\operatorname{Re}\{F(j \omega)\}$ using the angular frequency $\omega$ as a parameter. The same set of values for $A, B, \tau_{0}$ and $\tau_{1}$ as in the Eq. (13) was used to make the plot of Fig. 5. This Nyquist plot is used to make the fitting with the three layer thermal model.

Strictly speaking, the plot in Fig. 5 is the Nyquist plot of the experimentally measured surface temperature. In order to compare the experimental results with the three layer model, we should know the value of the power flux $P_{\mathrm{AC}}$. One should apply then a constant power flux at the surface and record the transient temperature rise. However, experimentally it is much easier to apply a previous cooling and to measure the skin temperature rise afterwards. From the fitting we will obtain a numerical value for $P_{\mathrm{AC}}$ which can be interpreted as the power flux which would give rise to the same skin temperature as the one observed experimentally.

On the one hand we have developed a three layer model which can easily provide a thermal response in the frequency domain. On the other hand experimental

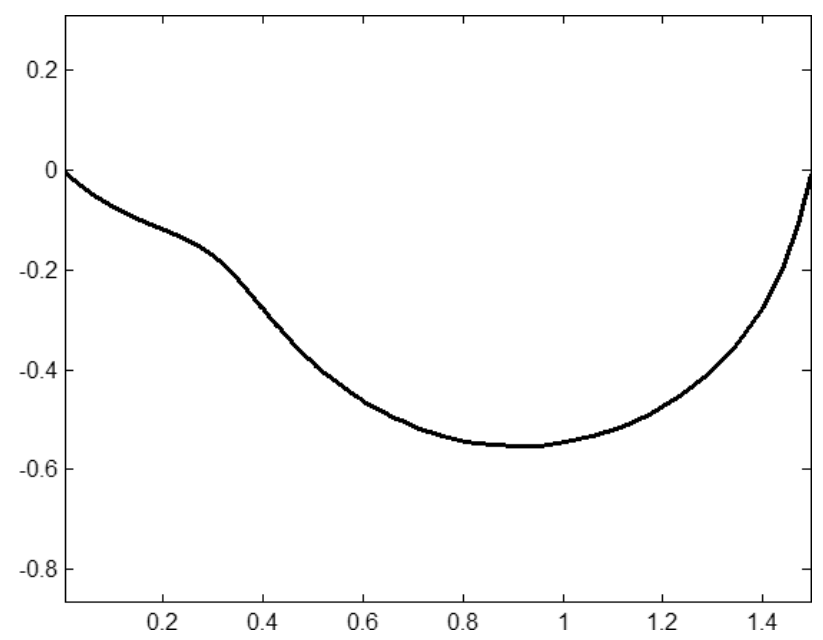

Fig. 4. Nyquist plot of the function $F(j \omega)$. 


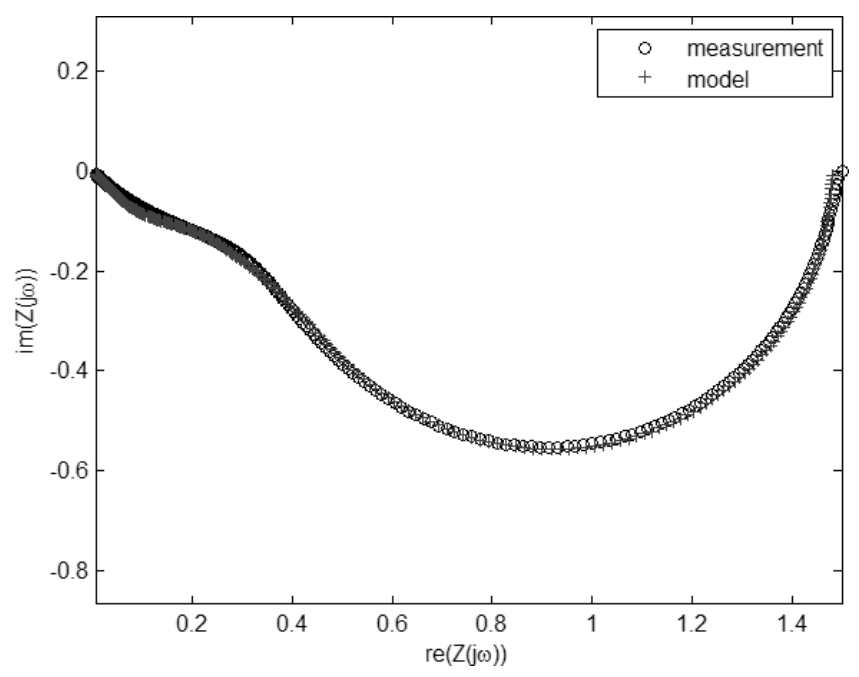

Fig. 5. Fitting the complex thermal impedance.

measurements have been carried out giving rise to a step response in the time domain. The latter one could be fitted very well to an analytical function (13), which after time derivation could be converted into the frequency domain in a form of complex thermal impedance. The final question is now: can the thermal impedance for the three layer model and for the measurements be fitted to each other? The fitting has been done not only by requiring both Nyquist plots to be coincident (Fig. 5), but it was also demanded that the three layer model and the function $F$ should have the same complex values for the same frequency. The results shown in Fig. 5 and Table 1 were obtained with a heat transfer coefficient $h=27.33 \mathrm{~W} / \mathrm{m}^{2} \mathrm{~K}$. It validates our model because we were able to obtain a theoretical curve using an acceptable set of parameters which could be very well fitted to a Nyquist plot obtained with thermographic measurements. Only the heat transfer coefficient $h=27.33 \mathrm{~W} / \mathrm{m}^{2} \mathrm{~K}$ turns out to be too high, but it must be remarked here that in the beginning of the measurement, the compressed air was blown for the skin cooling, which gives an inevitably rise to an enhanced heat transfer coefficient.

Table 1. Parameters' values for fitting modeling and measurement results.

\begin{tabular}{lcccc}
\hline $\begin{array}{l}\text { Material }(\rightarrow) \\
\text { Parameter }(\downarrow)\end{array}$ & $\begin{array}{c}\text { Layer } 1 \\
\text { epidermis }\end{array}$ & $\begin{array}{c}\text { Layer } 2 \\
\text { dermis }\end{array}$ & $\begin{array}{c}\text { Layer } 3 \\
\text { hypodermis }\end{array}$ & Blood \\
\hline Thermal conductivity $k$ & $k_{1}=0.127$ & $k_{2}=0.758$ & $k_{3}=0.830$ & - \\
$(\mathrm{W} / \mathrm{m} \cdot \mathrm{K})$ & $d_{1}=0.08$ & $d_{2}=1$ & $d_{3}=3$ & - \\
Thickness $d(\mathrm{~mm})$ & $\varrho_{1}=2000$ & $\varrho_{2}=2000$ & $\varrho_{3}=1845$ & $\varrho_{b}=800$ \\
Density $\varrho\left(\mathrm{kg} / \mathrm{m}^{3}\right)$ & $c_{1}=4000$ & $c_{2}=3657$ & $c_{3}=2444$ & $c_{b}=2000$ \\
Specific heat $c(\mathrm{~J} / \mathrm{kgK})$ & $w_{1}=1.010^{-8}$ & $w_{2}=0.0049$ & $w_{3}=0.0005$ & - \\
Perfusion $w(1 / \mathrm{s})$ & & & & \\
\hline
\end{tabular}


The values shown in Table 1 are all physically acceptable. It validates our model with the thermographic measurements. The fitting can be implemented automatically using optimization procedures as well.

\section{Conclusions}

In this contribution a three layer model has been presented for the temperature distribution in the human skin. It must be emphasized here that the model is a time dependent one. Using the Laplace domain, the equations could be easily solved. For the experimental part, a transient heating curve was recorded using infrared thermography. As the theoretical model was solved in the Laplace domain, a direct comparison was not possible. Therefore a new approach has been presented. First of all the transient temperature curve was fitted to an analytical function. This function could be exactly converted into the Laplace domain. It was possible to obtain a good fitting with acceptable values for all the thermal parameters of the three skin layers. In the next step of the research we will make the fitting of modeling and measurement results fully automatic and use the values of the thermal parameters to differentiate the physiological and pathological states of the skin.

\section{Acknowledgement}

This work was supported by the National Science Centre Poland, project no. DEC2013/11/N/ST7/02630.

\section{References}

1. Buzug TM, Schumann S, Pfaffmann L, Reinhold U, Ruhlmann J, Functional infrared imaging for skin-cancer screening, Conf Proc IEEE Eng Med Biol Soc 1:2766-2769, 2006.

2. Laaperi E, Laaperi A, Strakowska M, Wiecek B, Przymusiała P, Cold provocation improves breast cancer detection with IR thermography — a pilot study, Thermol Int 22:152-156, 2012.

3. Pennes HH, Analysis of tissue and arterial blood temperatures in the resting forearm, $J$ Appl Physiol 1:93-122, 1948.

4. Klinger H, Heat transfer in perfused biological tissue. I. General theory, Bull Math Biol 21:494-497, 1974.

5. Chen MM, Holmes KR, Microvascular contributions in tissue heat transfer, Ann New York Acad 335:137-150, 1980.

6. Weinbaum S, Jiji LM, Lemons DE, Theory and experiment for the effect of vascular microstructure on surface tissue heat transfer. Part I. Anatomical foundation and model conceptualization, ASME J Biomech Eng 106:321-330, 1984.

7. Weinbaum S, Jiji LM, A new simplified bioheat equation for the effect of blood flow on local average tissue temperature, ASME J Biomech Eng 107:131-139, 1985.

8. Zolfaghari A, Mehdi Maerefat M, Bioheat transfer, developments in heat transfer, Dr. Marco Aurelio Dos Santos Bernardes (Ed.), ISBN: 978-953-307-569-3, InTech, 2011, 
Available at: http://www.intechopen.com/books/developments-in-heat-transfer/bioheat-transfer.

9. Ng EYK, Tan H, Ooi E, Boundary element method with bioheat equation for skin burn injury, Burns 35:987-997, 2009.

10. Więcek B, Strạkowska M, De Mey G et al., Influence of infrared radiation on the human skin temperature - Experimental data and modeling, J Mech Med Biol 13(3):13500251-10, 2013.

11. Lin S-M, Analitical solution of bio-heat conduction on skin in Fourier and non-Fourier models, J Mech Med Biol 13(4):1350063-1-17, 2013.

12. Ruminski J, Kaczmarek M, Renkielska A, Nowakowski A, Thermal parametric imaging in the evaluation of skin burn depth, IEEE Trans Biomed Eng 54(2):303-312, 2007.

13. Nowakowski A, Kaczmarek M, Active dynamic thermography-Problems of implementation in medical diagnostics, QIRT J 8(1):89-106, 2011.

14. Tan H, Ng EYK, Acharya U, Chee C, Infrared thermography on ocular surface temperature: A review, Infrared Phys Technol 52:97-108, 2009.

15. Ng EYK, A review of thermography as promising non invasive detection modality for breast tumor, Int $J$ Thermal Sci 48:849-855, 2009.

16. Vermeersch B, De Mey G, Influence of substrate thickness on thermal impedance of microelectronic structures, Microelectron Reliabil 47:437-443, 2007.

17. De Mey G, Vermeersch B, Banaszczyk J, Świątczak T, Więcek B, Janicki M, Napieralski A, Thermal impedances of thin plates, Int J Heat Mass Transf 50:4457-4460, 2007.

18. Świạtczak T, Vermeersch B, De Mey G, Więcek B, Banaszczyk J, Felczak M, Evaluation of the heat transfer coefficient in microcircuits from the frequency analysis of the thermal response, IEEE Trans Components Pack Technol 6:260-266, 2010.

19. Kawka P, De Mey G, Vermeersch B, Thermal characterisation of electronics packages using the Nyquist plot of the thermal impedance, IEEE Trans Components Pack Technol 30:660-665, 2007.

20. Carslaw H, Jaeger J, Conduction of Heat in Solids, Clarendon Press, Oxford, 1971.

21. Cengel Y, Heat Transfer, McGraw Hill, Boston, 2003.

22. Bejan A, Heat Transfer, John Wiley \& Sons, New York, 1993, pp. 151-155.

23. Abramowitz M, Stegun I, Handbook of Mathematical Functions, Dover Publishing, New York, 1972. 